\title{
OCORRÊNCIA DE MIROUNGA LEONINA LINNAEUS (PINNIPEDIA, PHOCIDAE) NO EXTREMO-SUL DO BRASIL: AGRESSÃO E PATOLOGIAS
}

\author{
César Jaeger Drehmer ${ }^{1}$ \\ Jorge Ferigolo ${ }^{2}$ \\ Eduardo Sérgio Borsato ${ }^{2}$
}

\begin{abstract}
Occurrence of Mirounga leonina Linnaeus (Pinnipedia, Phocidae) FROM SOUTHERNMOST BRAZIL: INJURY AND PATHOLOGIES. A male specimen of Mirounga leonina Linnaeus, 1758 age estimated between 7-8 years old, collected at Santa Vitória do Palmar, Rio Grande do Sul State, southernmost Brazil $\left(32^{\circ} 44^{\prime}\right.$ S and $53^{\circ} 22^{\prime} \mathrm{W}$ ) is presented. All the skeleton was recovered except the rostral region. It shows an advanced osteomyelitis in the left dentary, extending from the synfisis until the middle portion of the body; as well as Scheuermann disease at lumbar vertebrae. Such diseases could explain its presence at that locality, where it was shot. The bullet was recovered from the rostrum, and might be responsible for death. This is the first virtually complete skeleton of $M$. leonina recovered from Brazilian coast.

KEY WORDS. Pinnipedia, Phocidae, Mirounga leonina, osteomyelitis, Scheuermann disease, injury
\end{abstract}

Nas últimas décadas tem sido dado destaque à ocorrência de espécies fora de suas áreas usuais de distribuição. No caso de mamíferos marinhos, tanto cetáceos quanto pinipédios, os registros são mais abundantes devido à ampla distribuição geográfica e à facilidade com que os animais se deslocam pelos oceanos.

SIMÕES-LOPES et al. (1995) apresentaram uma ampla revisão das ocorrências de otarídeos e focídeos para a costa norte do estado do Rio Grande do Sul e para a costa de Santa Catarina, apresentando novas ocorrências para Mirounga leonina Linnaeus, 1758 baseadas em avistagens e registro fotográfico. Segundo LING \& BRYDEN (1981), a distribuição desta espécie é circumpolar antártica, desde $78^{\circ} \mathrm{S}$, mas também podem alcançar $16^{\circ} \mathrm{S}$ (Ilha de Santa Helena) no Atlântico. Os locais de reprodução concentram-se entre 40 e $62^{\circ} \mathrm{S}$ nos Oceanos Índico e Atlântico, sendo considerados "indivíduos ocasionais" os que chegam ao litoral do Uruguai e do Brasil (LING \& BRYDEN 1981), como resultado de "movimentos erráticos".

No Brasil já foram avistados indivíduos em algumas localidades. LODI \& SICILIANO (1989) registraram um indivíduo, provavelmente macho e em estágio de muda, a $3^{\circ} 52^{\prime}$ 'S e $32^{\circ} 28^{\prime} \mathrm{W}$, no Arquipélago de Fernando de Noronha. Um outro indivíduo foi avistado na praia do Tororão, município de Prado, Bahia, aproxima-

1) Departamento de Zoologia, Instituto de Biologia, Universidade Federal de Pelotas. Campus Universitário, Caixa Postal 354, 96010-900 Pelotas, Rio Grande do Sul, Brasil.

2) Museu de Ciências Naturais, Fundação Zoobotânica do Rio Grande do Sul. Avenida Salvador França 1427, Caixa Postal 1188, 90690-000 Porto Alegre, Rio Grnade do Sul, Brasil. 
damente a $17^{\circ} 25^{\prime}$ 'S e $39^{\circ} 15^{\prime} \mathrm{W}$ (R.V. Carvalho comunicação pessoal). Além destes, há também registros para o Brasil meridional. PINEDO (1990) apresentou uma relação das ocorrências referindo, além dos registros acima, ocorrências para o Rio de Janeiro, Paraná, Santa Catarina e Rio Grande do Sul. De um total de dez ocorrências, apenas o crânio de 1 espécime foi coletado, de um macho, na praia do Cassino, município de Rio Grande, Rio Grande do Sul, depositado na coleção do Museu Oceanográfico de Rio Grande. Recentemente foi recuperado no município de Mostardas, Rio Grande do Sul, parte do esqueleto de um elefante-marinho (P.H. Ott comunicação pessoal).

\section{MATERIAL E MÉTODOS}

O material pertence à Coleção de Mastozoologia do Museu de Ciências Naturais da Fundação Zoobotânica do Rio Grande do Sul, Porto Alegre, sob o número MCN 2768. A preparação osteológica está referida em DREHMER \& FERIGOLO (1996a,b). O exame de balística foi efetuado na Seção de Balística Forense do Instituto de Criminalística do Rio Grande do Sul.

A osteometria do espécime esta apresentada na tabela I. Dos ossos assinalados com asterisco $(*)$ foram tomadas medidas apenas do corpo do osso sem considerar as epífises. A craniometria não foi efetuada pela ausência da região rostral, comprometendo as medições.

Tabela I. Osteometria de Mirounga leonina (MCN 2768). Comprimento em milímetros dos principais ossos.

\begin{tabular}{lccc}
\hline \multicolumn{1}{c}{ Ossos } & Direito & Esquerdo & Heterotópico \\
\hline Báculo & - & - & 366,2 \\
Escápula & $333,6^{*}$ & $335,7^{*}$ & - \\
Fêmur & 169,1 & 169,2 & - \\
Inominado & $363,6^{*}$ & $363,3^{*}$ & - \\
Rádio & 307,0 & 309,5 & - \\
Tíbia e fíbula & 414,5 & 418,1 & - \\
Ulna & $299,4^{*}$ & $302,3^{*}$ & - \\
Úmero & 266,6 & 274,6 & - \\
\hline
\end{tabular}

*) Sem epífise.

Abreviaturas utilizadas no texto: $(\mathrm{C})$ vértebras cervicais; $(\mathrm{Cd})$ vértebras caudais; (GLG) growth layer groups; (L) vértebras lombares; (L1) primeira vértebra lombar; (L2) segunda vértebra lombar; (L3) terceira vértebra lombar; (MCN) Museu de Ciências Naturais; $(\mathrm{S})$ vértebras sacrais; $(\mathrm{T})$ vértebras torácicas.

\section{RESULTADOS E DISCUSSÃO}

Data e local. 05 de julho de 1991 , a $32^{\circ} 44^{\prime} \mathrm{S}$ e $53^{\circ} 22^{\prime} \mathrm{W}$, no município de Santa Vitória do Palmar, Rio Grande do Sul, extremo-sul do Brasil, a aproximadamente $400 \mathrm{~m}$ da divisa com o Uruguai. 
Esqueleto. Do crânio estão preservados os frontais, parietais e o occipital em vista dorsal (Fig. 1), e a porção posterior dos palatinos, o vômer, o basisfenóide, os pterigóides, os temporais (bulas timpânicas, fossas mandibulares e processos zigomáticos dos esquamosais) e o occipital em vista ventral (Fig. 2). A fórmula vertebral encontrada foi C7 T15, L5 S3 Cd11.
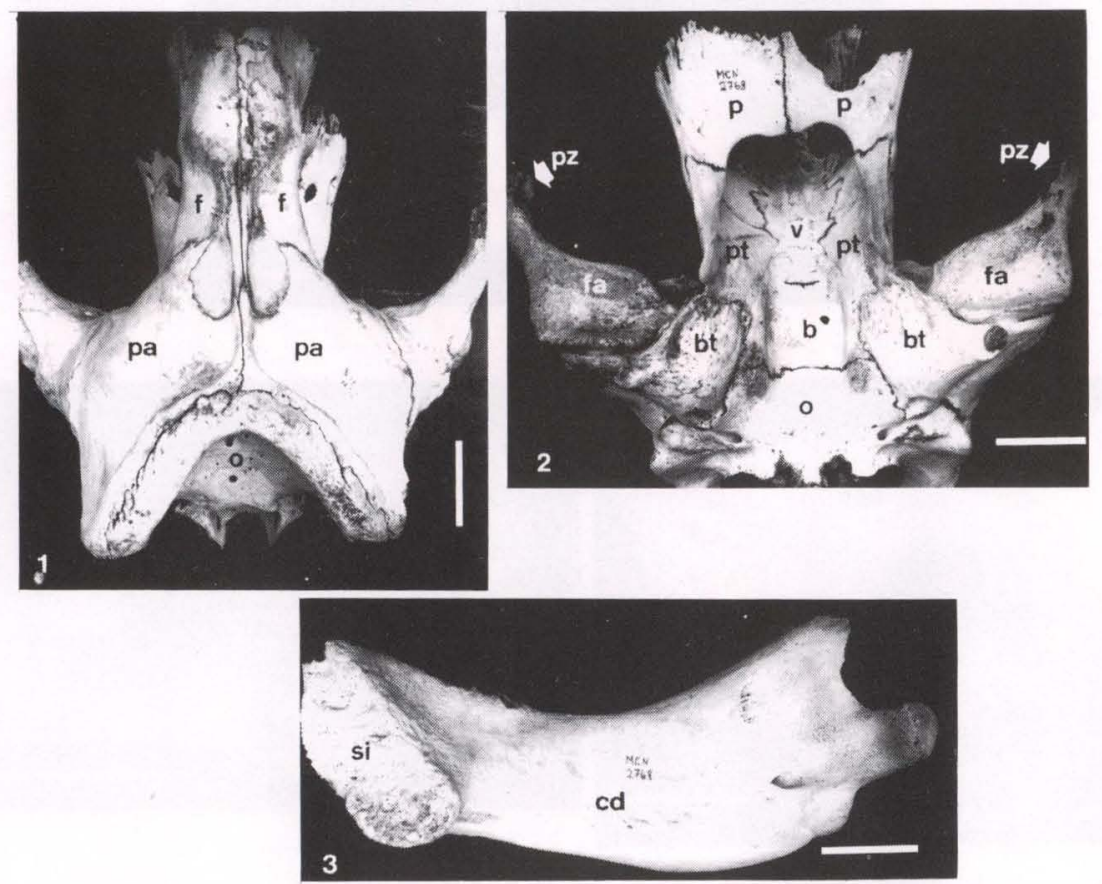

Figs 1-3. Sincrânio de M. leonina (MCN 2768). (1) Vista dorsal do crânio; (2) vista palatal do crânio; (3) vista medial do dentário direito (canino removido). (b) Basisfenóide, (bt) bula timpânica, (cd) corpo do dentário, (f) frontal, (fa) fossa mandibular, (o) occipital, (p) palatino, (pa) parietal, (pt) pterigóide, (pz) processo zigomático do esquamosal, (si) sínfise, (v) vômer. Barra $=50 \mathrm{~mm}$.

Sexo e idade. Macho, de aproximadamente $390 \mathrm{~cm}$ de comprimento. As medidas corporais foram estimadas através do registro fotográfico. Devido à ausência da região rostral do crânio (Figs 1-2), foi impossível estimar a idade do animal pelos métodos tradicionais de comprimento côndilo-basal ou de comprimento basilar (BRIGGS \& MOREJOHN 1976), ou de suturas cranianas (KING 1972). Igualmente a contagem de GLG's não foi efetuada a fim de não danificar os dentes caninos (Figs 7-8). Entretanto, segundo os dados de crescimento para indivíduos das populações da Ilha Geórgia do Sul (LING \& BRYDEN 1981), este indivíduo possuía entre 7 e 8 anos. Muito provavelmente o espécime proveio das populações das Ilhas Malvinas $\left(52^{\circ} \mathrm{S}, 58-62^{\circ} \mathrm{W}\right)$ e adjacências, onde se incluem as Ilhas Geórgia do Sul, ou das colônias continentais como as da Península Valdéz, Argentina (aproximadamente $42^{\circ} \mathrm{S}$ e $64^{\circ} \mathrm{W}$ ) já que as outras duas grandes concentrações 
populacionais localizam-se em ilhas muito mais distantes (Crozet e Macquarie). Considerando que os machos desta espécie estão sexualmente maduros entre 4 e 6 anos (BRIGGS \& MOREJOHN 1976), este espécime pode ser considerado fisiologicamente um adulto, mas a rigor não estabelecera uma colônia reprodutiva.
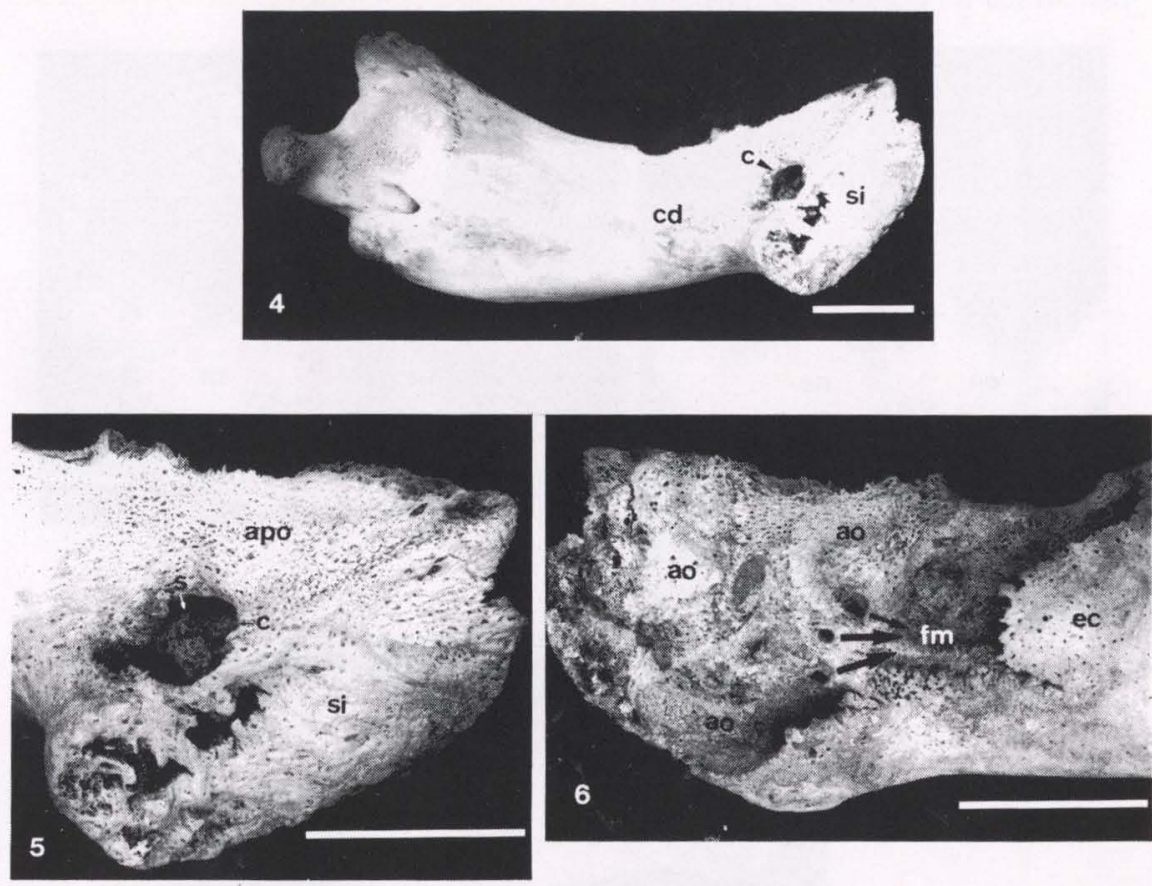

Figs 4-6. Dentário esquerdo de M. leonina (MCN 2768). (4) Vista medial; (5) vista medial, detalhe da região sinfisial; (6) vista lateral, detalhe da região anterior. (ao) Área de osteólise, (apo) área de porose óssea, (c) cloaca, (cd) corpo do dentário, (ec) expansão da cortical, (fm) forames mentonianos, (s) seqüestro, (si) sínfise. Barra $=50 \mathrm{~mm}$.

Agressão (Figs 8, 10). Foi encontrado um projétil na porção anterior do crânio que apresenta um núcleo de liga endurecida de chumbo e jaqueta de latão, pesando 4,6 g, com $1,18 \mathrm{~cm}$ de comprimento e $0,78 \mathrm{~cm}$ de diâmetro na base, e ainda 5 ressaltos e 5 cavados dextrógiros, gravados em sua superfície cilíndrica. Foi expelido pelo cano de uma pistola 7,65 $\mathrm{mm}$ (.32 Auto), com cinco raias internas orientadas à direita, ou por um revólver calibre 32, com cano de cinco raias dextrógiras. A possibilidade do animal ter sido alvejado post-mortem está descartada, pela destruição total da região rostral que não pode ser recuperada. Apresenta-se uma reconstituição do projétil (Fig. 10).

Patologias (Figs 4-9, 11-15). O espécime apresenta osteomielite crônica no dentário esquerdo desde a região sinfisiária até a porção média do corpo do dentário (Fig. 4). Em vista medial, na metade proximal da goteira lingual existe uma extensa cloaca aproximadamente circular $(3,5 \mathrm{~cm} \times 2,5 \mathrm{~cm})$, através da qual observa-se um 

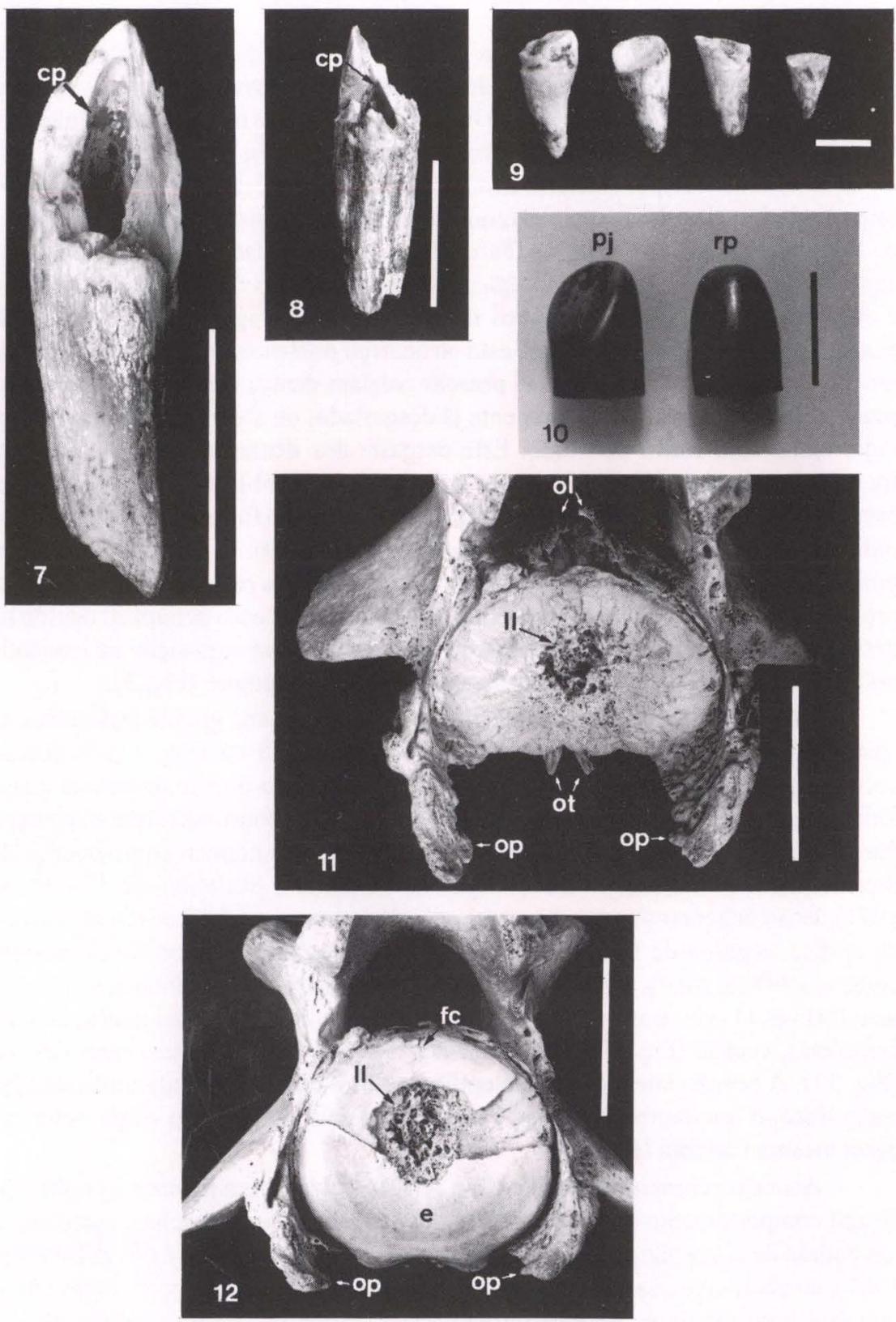

Figs 7-12. M. leonina (MCN 2768). (7) Vista lingual do canino direito; (8) vista lingual do canino esquerdo; (9) Vista medial dos pós-caninos fraturados; (10) projétil e reconstituição; (11) vista posterior da L3 (epífise removida); (12) vista posterior da L3 com parte da epífise. (cp), Cavidade pulpar, (e) epifise, (fc) fratura consolidada, (II) lesão lítica, (ol) ossificações ligamentares, (op) ossificações paravertebrais, (ot) osteófitos, (pj) projétil, (rp) reconstituição do projétil. Barra $=10 \mathrm{~mm}$ (Figs 9, 10); barra $=50 \mathrm{~mm}$ (Figs 7, 8, 11, 12). 
grande seqüestro de formato irregular, com cerca de $3 \mathrm{~cm}$ de comprimento (Fig. 5). $\mathrm{Na}$ região sinfisial existem ainda duas possíveis cloacas menores de formato irregular, e na metade distal da goteira lingual, bem como na região ventral à margem alveolar, existe uma área de forte porose óssea (Fig. 5). Na face lateral existe uma expansão da cortical, por extensão da osteomielite sub-corticalmente, e também uma importante área de osteólise, envolvendo toda a região anterior do dentário, inclusive os forames mentonianos (Fig. 6). Observa-se ainda osteólise na base do alvéolo do canino esquerdo de modo que o seqüestro é visível através dela. Os caninos direito e esquerdo encontram-se fraturados na base da coroa (Figs 7-8). A ausência de desgaste nestas fraturas indica que esta ou ocorreu post-mortem, ou pouco antes da morte do indivíduo. Como muitas pessoas coletam dentes dos animais mortos na praia, o mais provável é que um dente já desgastado, ou até mesmo fraturado, em vida, tenha sido assim destruído. Este desgaste dos dentes em vida ocorre com freqüência em pinipédios (DREHMER \& FERIGOLO 1996b), como é o caso deste espécime, onde alguns dentes pós-caninos inferiores estão fraturados e desgastados, indicando que essas fraturas ocorreram em vida (Fig. 9). Considerando que em pinipédios machos em geral são comuns fraturas dentárias resultantes de combates, provavelmente a osteomielite foi complicação de um abscesso periapical devido ao desgaste ou até mesmo à fratura do canino esquerdo com exposição da cavidade pulpar (Fig. 8). O dentário direito não apresenta tais patologias (Fig. 3).

No corpo da terceira vértebra lombar observa-se uma grande lesão lítica na epífise posterior de formato circular e com diâmetro de $3 \mathrm{~cm}$ (Fig. 11). A porção correspondente à lesão está ausente na epífise, entretanto o resto desta está quase todo preservado. Esta epífise apresenta ainda uma fratura consolidada em sua porção dorsal (Fig. 12). Lesões quase idênticas são descritas no homem como doença de Scheuermann (="Cifose juvenil", "Epifisite vertebral"; SCHMORL \& JUNGHANS 1971), lesão degenerativa pós-traumática do disco inter-vertebral intrassomático, e da epifise, seguida de lesões intrassomáticas. Na região posterior da L3 existem ainda osteófitos, ossificações paravertebrais e ossificações ligamentares sob o arco neural (Figs 11-12). Entre L1 e L2 tais ossificações formam pontes intervertebrais completas, ventral (Fig. 13) e lateralmente (Fig. 14), e incompletas, ventralmente (Fig. 13). A porção látero-dorsal da epífise anterior da L2 apresenta uma pequena lesão lítica, o que também se observa em uma pequena região do corpo vertebral desta mesma vértebra (Fig. 15).

Aspectos comportamentais. Com relação à Mirounga leonina é conhecido de seu comportamento que alguns indivíduos, especialmente machos, apresentam um padrão de dispersão ao acaso ao invés de migrações em massa (LING \& BRYDEN 1981); constatou-se que há exclusão de indivíduos machos do processo reprodutivo (em que pese os meses de inverno não serem de reprodução) e também há alta capacidade de deslocamento em pinipédios altamente dimórficos sexualmente, sobretudo machos (BARTHOLOMEW 1970). Acrescenta-se que a presença deste indivíduo na costa do Brasil poderia relacionar-se ao fato conhecido de animais doentes se afastarem do convívio dos seus semelhantes como ocorre em outras espécies de grandes mamiferos (elefantes, búfalos e cervídeos). 

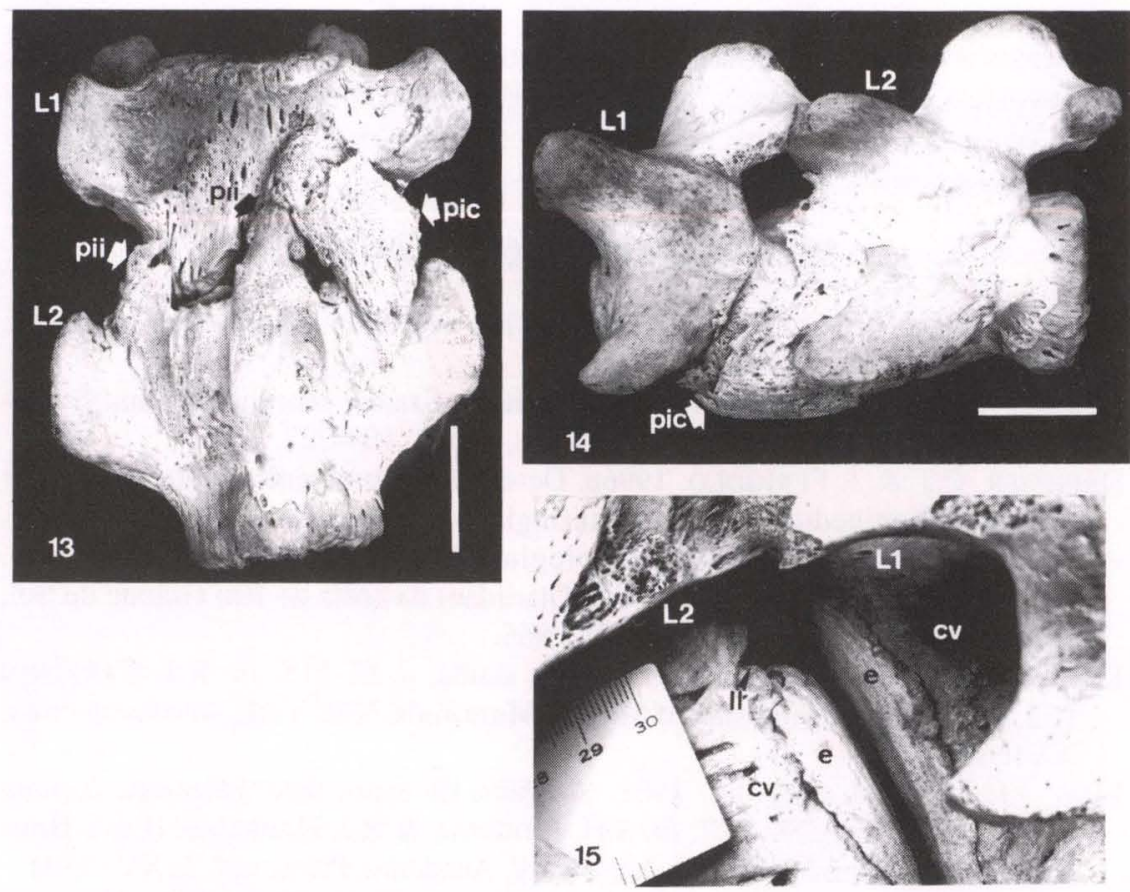

Figs 13-15. L1 e L2 de M. leonina (MCN 2768). (13) Vista ventral; (14) vista lateral; (15) detalhe em vista dorsal da região intervertebral. (cv) Corpo vertebral, (e) epífise, (L1) primeira vértebra lombar, (L2) segunda vértebra lombar, (II) lesão lítica, (pic) ponte intervertebral completa, (pii) pontes intervertebrais incompletas. Barra $=50 \mathrm{~mm}$, exceto figura 15.

\section{CONCLUSÕES}

Registrou-se a presença de um espécime macho da espécie Mirounga leonina em $32^{\circ} 44^{\prime}$ S e $53^{\circ} 22^{\prime} \mathrm{W}$, no município de Santa Vitória do Palmar, Rio Grande do Sul, extremo sul do Brasil, fronteira com o Uruguai, no qual constatou-se: a) osteomielite mandibular crônica que certamente comprometia a capacidade de alimentação do indivíduo; b) fratura em vida de alguns dentes pós-caninos; c) infecção provavelmente devida a abscesso periapical relacionado ao desgaste ou fratura do canino esquerdo; d) provável doença de Scheuermann em vértebras lombares, comprometendo significativamente a capacidade de deslocamento do indivíduo o que pode ter favorecido seu "movimento errático" até a costa do Brasil; e) o projétil recuperado, por suas características físicas, é componente de munição de arma de fogo de calibre 7,65 Browning ou.32 Auto; f) embora doente, o indivíduo deve ter sido morto, muito próximo ou mesmo na costa do Brasil, em conseqüência das lesões sofridas pelo disparo que $o$ atingiu.

AGRADECIMENTOS. Ao CNPq, através do seu programa RHAE (Bolsa DTI, autor sênior) e a Fundação Zoobotânica do Rio Grande do Sul pelo suporte financeiro para as coletas. $\grave{A}$ 
FAPERGS que possibilitou a divulgação (Processo n 97/1736-5). Aos colegas, José Luciano Maciel pelo auxílio na confecção das figuras, e Fernando S. Stobbe pelo auxílio na preparação do espécime. À bióloga Maria Lúcia Machado e ao Perito Criminalístico Leonardo Alves Graziuso pela análise balística.

\section{REFERÊNCIAS BIBLIOGRÁFICAS}

BARTHOLOMEW, G.A. 1970. A model for the evolution of pinniped polygyny. Evolution (24): 546-559.

Briggs, K.T. \& V. MoreJohn. 1976. Dentition, Cranial Morphology and Evolution in Elephant Seals. Mammalia, Paris, 40 (2): 199-222.

Drehmer, C.J. \& J. Ferigolo. 1996a. Descrição do sincrânio de Arctocephalus australis (Pinnipedia, Otariidae). Iheringia, Sér. Zool., Porto Alegre, 81: 63-74.

- 1996b. Anomalias e patologias dentárias em Arctocephalus G. Saint-Hilaire \& Cuvier (Pinnipedia, Otariidae) da costa do Rio Grande do Sul, Brasil. Revta bras. Zool. 13 (4): 857-865.

KING, J.E. 1972. Observations on phocids skulls, p. 81-115. In: R.J. HARRISON (Ed.). Functional Anatomy of Marine Mammals. New York, Academic Press, XVII+451p.

LING, J.K. \& M.M. BRYden. 1981. Southern Elephant Seal Mirounga leonina Linnaeus, 1758, p.297-327. In: S.H. RIDGWAY \& R.J. HARRISON (Eds). Handbook of Marine Mammals. New York, Academic Press, vol. 2, XV+359p.

Lodi, L. \& S. Siciliano. 1989. A Southern Elephant Seal in Brazil. Mar. Mammal Sci. 5 (3): 313.

PINEDO. M.C. 1990. Ocorrência de pinípedes na costa brasileira. Garcia de Orta, Sér. Zool., Lisboa, 15 (2): 37-48.

SchmorL, G. \& H. Junghans. 1971. The Human Spine in Health and Disease. New York, Grune \& Stratton, $2^{\text {nd }}$ ed., XI+504p.

SimÕES-LoPES, P.C.; C.J. DREHMER \& P.H. OTT. 1995. Nota sobre os Otariidae e Phocidae (Mammalia, Carnivora) da costa norte do Rio Grande do Sul e Santa Catarina, Brasil. Biociências, Porto Alegre, 3 (1): 173-181.

Recebido em 12.IX.1997; aceito em 27.XI.1998 